\title{
Liver Lesions Detection and Classification in Ultrasound Images Using Gabor Characterization, Edge Detection and Artificial Neural Networks
}

\author{
Type of article: conference abstract
}

\author{
Naghme Dashti1*, Elias Ameli Bafandeh2 \\ 1: Master of Software Engineering, Technical and Vocational Training Organization, \\ Tehran, Iran \\ 2: Master of Software Engineering, Islamic Azad University, Mashhad, Iran \\ * Tel: +98 915102 5342, E-mail: Naghme.Dashti@gmail.com
}

\author{
ABSTRACT
}

Introduction: In the last decade one of the main reasons for people mortality and disability is liver diseases. Early detection of these diseases can help adopt appropriate treatment methods. Ultrasound imaging is a non-invasive method for visualizing tissue specification and liver lesions detection which its resolution is lower than CT and MRI images. Precise determination of liver tissue lesions and progression degree of disease is possible with advanced computer techniques such as artificial neural networks (ANN) from medical images. In this paper, a classification-based method is presented to identify and diagnose liver lesions using the Gabor wavelet features and edge detection. In this method, the vector of features from healthy and damaged tissues is trained to the network based on Gabor filters. Then the suspected cases of tissue lesions in various liver diseases are identified by features extraction of entry images. After that, the edge detection technique is implemented and the internal points of the edge are tested as an inputs of a neural network which determine the healthy and unhealthy liver tissues.

Methods: Image features are extracted and processed by Gabor wavelet. Also the ANN is used to liver disease classification based on the images features. The forward multilayer perceptron neural network is organized with three layers of input, hidden and output. The training of this network is done with back propagation method and all of the data include "healthy tissues" and "damaged tissues" of the liver are collected in a large cellular array. Furthermore, an edge detection technique is used to indicate the points where the intensity of the light changes sharply. The sharp changes in image characteristics are usually representative of important events and changes in environments characteristics.

Results: The results of the implementation indicate a significant reduction in processing time of liver ultrasound images and also increase the precision and accuracy of liver lesions detection (approximately 5\%) among different classified groups of hepatic patients compared with the similar image processing methods. In the proposed method, the total time of operations include feature extraction, image processing, lesions detection and diagnosis of the disease has been decreased by reduction of the number of examined points. In addition, an edge detection technique had been used to diagnose the size of damaged tissues in various liver diseases, which helps improve the early detection of tissue lesions because of reduction of the checking domain of points.

Conclusion: In this paper, a new method was presented to identify liver tissue lesions. Gabor wavelet method is employed to extract the features of the liver ultrasound images. These wavelets provide the context to understand the images frequency and their analysis in the area of the space, and given their great advantage, which is slow changes in the frequency domain, it is an appropriate filter to extract the image features. Then, the extracted features of the ultrasound images of various liver patients are stored to train a neural network, and finally the image processing method is performed to identify the healthy and damaged tissues and also to diagnose the type of disease. The search scope of problem is minimized as the input of the neural network to find the liver damaged tissue by the edge detection technique which is lead to errors reduction in identifying the tissue damages, increasing the detection speed of these lesions, and diagnosing the disease as well as determining the damage degree of liver.

KEYWORDS: Image Processing, Gabor Filter, Edge Detection, Artifitual Neural Network, Liver Lesion Detection 
Medical Technologies Journal, Volume: 1, Issue: 4, October-December 2017, Pages:76-136. Doi : https://doi.org/10.26415/2572-004X-vol1iss4

\section{Declaration of conflicts}

This abstract is selected from the First International Congress of Diseases and Health Outcomes Registry and First National Congress of Medical Informatics, 14-17 February 2017, Mashhad, Iran

\section{Authors' biography}

No biography.

\section{References}

No references. 